\title{
Evaporation-Induced Crystallization of Surfactants in Sessile Multicomponent Droplets
}

\author{
Yaxing Li, Valentin Salvator, Herman Wijshoff, Michel Versluis, and Detlef Lohse* \\ Cite This: Langmuir 2020, 36, 7545-7552 \\ Read Online
}

ABSTRACT: Surfactants have been widely studied and used in controlling droplet evaporation. In this work, we observe and study the crystallization of sodium dodecyl sulfate (SDS) within an evaporating glycerol-water mixture droplet. The crystallization is induced by the preferential evaporation of water, which decreases the solubility of SDS in the mixture. As a consequence, the crystals shield the droplet surface and cease the evaporation. The universality of the evaporation characteristics for a range of droplet sizes is revealed by applying a diffusion model, extended by

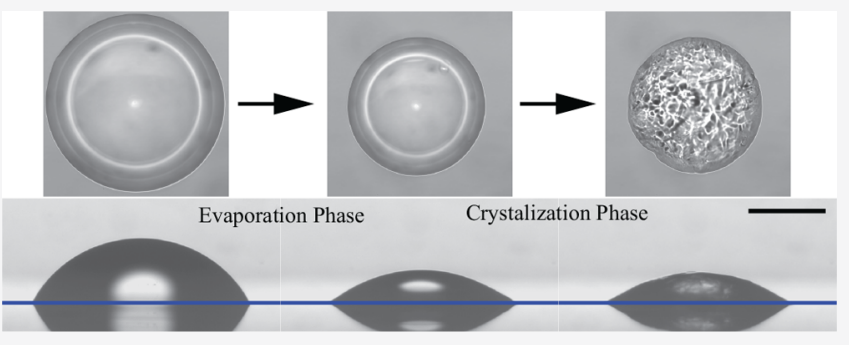
Raoult's law. To describe the nucleation and growth of the crystals, we employ the 2-dimensional crystallization model of Weinberg [J. Non-Cryst. Solids 1991, 134, 116]. The results of this model compare favorably to our experimental results. Our findings may inspire the community to reconsider the role of high concentration of surfactants in a multicomponent evaporation system.

\section{INTRODUCTION}

Surfactants are widely used to control the evaporation behavior of sessile droplets on a flat substrate. ${ }^{1-3}$ The motivation is driven by various applications in inkjet printing, surface coating, and patterning, ${ }^{4,5}$ which mainly aim to optimize the drying rate and the final deposition. The biggest challenge for a controlled uniform coating by droplet evaporation originates from the well-known "coffee-stain effect". ${ }^{6}$ It has been shown that surfactant-induced Marangoni flow can play an essential role to suppress this effect. ${ }^{7,8}$ In these studies, one of the most common ionic surfactants, "sodium dodecyl sulfate" (SDS), ${ }^{9-11}$ is added to the system at small concentration, typically $\leq 1$ wt $\%$. The surfactants are therefore considered to be always soluble in the system during most of the evaporation lifetime. However, in many practical cases, the relevant liquids contain a high concentration of surfactants; for example, liquid detergents can contain surfactant ingredients at up to $40 \mathrm{wt} \%$. Such a high loading of surfactants may lead to undesired effects, such as separation and crystallization.

Sodium dodecyl sulfate (SDS) may crystallize in liquid solutions upon cooling ${ }^{12}$ or upon seeding with 1-dodecanol. ${ }^{13}$ On the other hand, selective evaporation of some liquid components with larger volatilities can also lead to phase separation in multicomponent mixtures. ${ }^{14-16}$ Consequently, the nonvolatile surfactant (SDS) is expected to separate from an evaporating liquid system by crystallization due to the preferential evaporation of volatile liquids. Therefore, the wide usage of SDS in evaporating droplet systems deserves a more detailed explanation of the crystallization behavior.

In this work, we study a multicomponent droplet system consisting of a mixture of glycerol, water, and SDS and let it evaporate in ambient air. SDS is not miscible with pure glycerol, but it does dissolve in glycerol-water mixtures for large enough water concentration ratios. This behavior qualitatively resembles the ternary "ouzo" system ${ }^{17}$ consisting of water, ethanol, and anise oil, which nucleates in droplets for low enough ethanol concentrations. Tan et al. ${ }^{18,19}$ triggered this emulsification threshold by the selective evaporation of ethanol in an evaporating ouzo droplet. Similarly, the varying solubility of SDS in glycerol-water binary systems may also lead to phase separation due to the concentration change caused by the selective evaporation of water alone. In contrast to crystallization by cooling, ${ }^{12,13}$ here the oversaturation with SDS and the subsequent nucleation and growth of SDS crystals are caused by the preferential evaporation of water at room temperature. $^{20}$

To better understand the evaporation-induced crystallization in the mixture droplet system, two main questions need to be addressed: how does a surfactant-laden mixture droplet evaporate, and how does one model the crystallization during the evaporation? In this paper we want to answer these questions. A typical snapshot of an evaporating droplet is shown in Figure 2, where the two life phases can be distinguished: the evaporation phase and the crystallization

Received: April 22, 2020

Revised: June 1, 2020

Published: June 15, 2020 

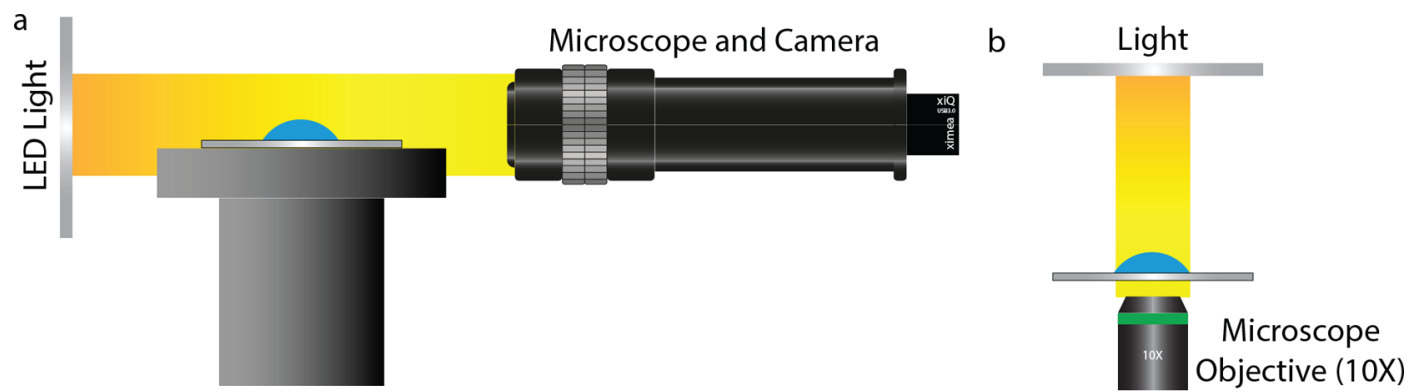

Figure 1. Schematic sketch of the experimental setups. (a) The contact angle device contains a CCD camera with a microscope and a LED light source illuminating the droplet. (b) The droplet is illuminated from above and recorded by a camera equipped with a $10 \times$ microscope objective underneath. The whole setup is part of a confocal microscope (Nikon A1 confocal laser microscope system, Nikon Corporation, Tokyo, Japan).

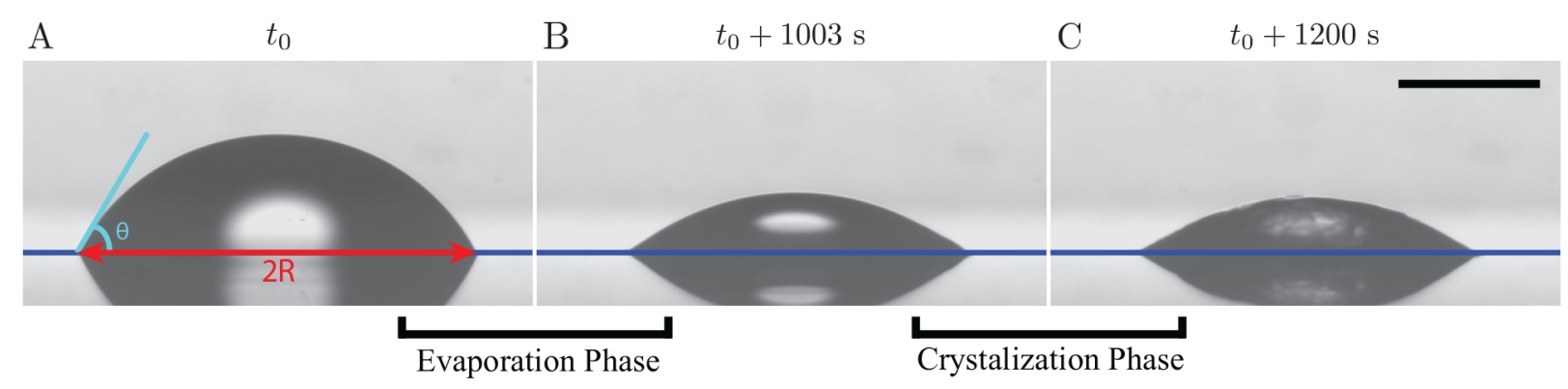

Figure 2. Experimental snapshots of the evaporation and drying process of a typical drop on a flat surface. (A) and (B) show the evaporation phase of the drop: here, the drop retains a spherical cap shape; no crystallization occurs. (C) The final state of the drop: because of the crystallization of SDS in the bulk, the surface of the drop buckles and no longer remains spherical. The crystallization of the SDS shields the surface and brings the evaporation process to an end. The scale bar represents $0.5 \mathrm{~mm}$.

phase. The focus of our study is on the dynamics of the evaporation and the kinetics of crystallization and not on the microscale crystal morphology.

\section{EXPERIMENTAL METHODS}

Materials and Preparation. The liquid solution was prepared with an initial composition of $78 \%(\mathrm{w} / \mathrm{w}$ ) Milli-Q water (Reference A +, Merck Millipore, $\left.25^{\circ}\right), 19.6 \%(\mathrm{w} / \mathrm{w})$ glycerol (Sigma-Aldrich; purity $\geq 98 \%$ ), and $2.4 \%(\mathrm{w} / \mathrm{w})$ sodium dodecyl sulfate (SigmaAldrich, purity 98\%). The initial concentration of SDS is 13CMC (critical micelle concentration). Experiments were performed on a transparent hydrophobic octadecyltrichlorosilane (OTS)-glass substrate. $^{21}$ The static contact angles of Milli-Q water and glycerol on the substrate are $105 \pm 3^{\circ}$ and $90 \pm 3^{\circ}$, respectively. The glycerol-water binary droplet with $50 \% / 50 \%(\mathrm{w} / \mathrm{w})$ has a $95^{\circ}$ static contact angle. Prior to each experiment, the samples were cleaned by sonication in an ultrasonic bath of ethanol and subsequently in water and then dried under a flow of nitrogen gas.

Experimental Setup. We performed two different experiments to separately study the evaporation phase and crystallization phase. To study the evaporation behavior, the droplets were deposited on the substrate by a Hamilton $2 \mu \mathrm{L}$ syringe, which was mounted vertically on a computer-controlled motorized pump, which allowed the dispensing of droplets of a controlled volume through a needle. We measured the geometry of the deposited droplet by bright-field imaging in side view. The whole process was recorded by an OCA 15 (Dataphysics, Germany) contact angle device (Figure 1a): a CCD camera coupled to a microscope, which was back-illuminated by a LED light from the opposite side of the droplet. For the crystallization study, we observed the droplet in bottom view with a confocal microscope (Figure 1b). By focusing on the layer close to the substrate (at $a \approx 10 \mu \mathrm{m}$ height), the dynamic growth of the crystals was visualized in a 2-dimensional view. The experiments were performed at room temperature of $21.4 \pm 1{ }^{\circ} \mathrm{C}$ and at relative humidity of $50 \pm 5 \%$. These parameters were monitored and recorded for each measurement.
Imaging Analysis. For the side-view geometrical measurement, images were analyzed by using a custom-made Matlab code to detect the droplet profile with subpixel accuracy. ${ }^{22}$ The sizes of all droplets are smaller than the capillary length $\sqrt{\gamma /(\rho g)} \approx 2.7 \mathrm{~mm}$ for the used liquids, ${ }^{23}$ where $\gamma \approx 70 \mathrm{mN} / \mathrm{m}$ and $\rho \approx 10^{3} \mathrm{~kg} / \mathrm{m}^{3}$ are the surface tension and density of the mixture and $g=9.8 \mathrm{~m} / \mathrm{s}^{2}$ is the gravitational acceleration. The detected profile is fitted to a spherical cap during the evaporation phase, which enables us to calculate the volume $V$ of the droplet with footprint radius $R$ and contact angle $\theta$. As shown in Figure 2A, the dark blue solid line is the position of the substrate: the spherical shape above it is the sessile droplet, the one underneath is its reflection.

For the documentation of the crystallization process from the bottom view, a manual detection with ImageJ was used to measure the crystallized area at every time instant; see details in the Supporting Information.

\section{RESULTS AND DISCUSSION}

Evaporation Phase. The left column of Figure 3 displays the temporal evolution of the drop-characterizing geometrical parameters for four droplets with different initial sizes: volume $V$ (A1), contact angle $\theta(\mathrm{B} 1)$, and footprint radius $R(\mathrm{C} 1)$. From the plots, it is evident that all the droplets evaporate following the "stick-slide" mode, ${ }^{24,25}$ in which the droplet's footprint radius first remains constant until it reaches a critical contact angle, and then the contact line starts to shrink. We only measure the volume until buckling occurs (as marked by the red circles in Figure 3A1,C1), and after that the droplet shape deforms and no regular shape is reestablished, which from then on renders accurate volume measurement impossible. Figure 4A shows the average evaporation rate of various droplets in the first $30 \mathrm{~s}$ after deposition with initial volumes ranging from 0.12 to $2.40 \mu \mathrm{L}$. The evaporation rate 

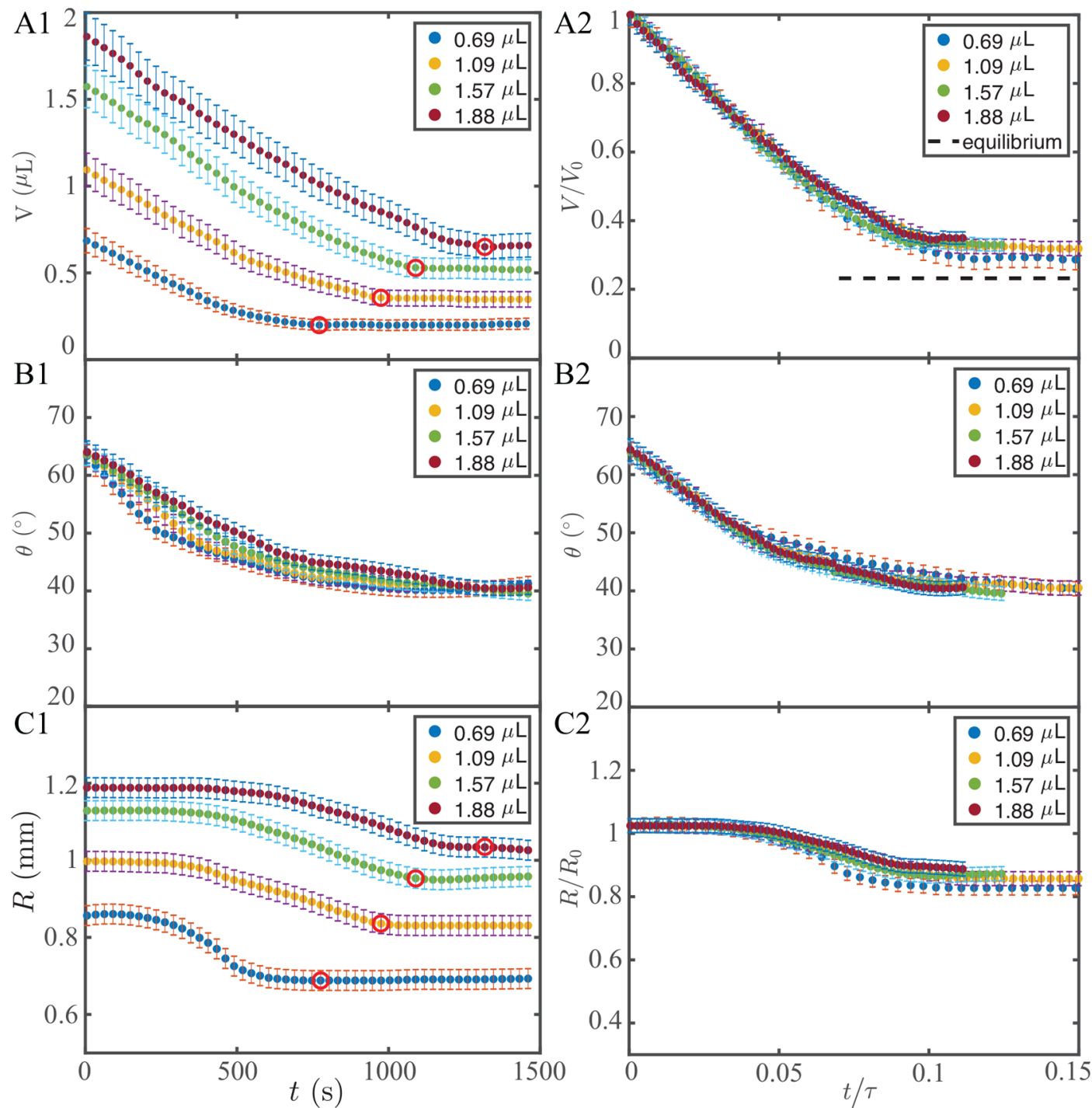

Figure 3. (A1, B1, C1) Measured temporal evolution of the geometrical parameters: volume $V$ (A1), contact angles $\theta$ (B1), and lateral sizes $R$ (C1). The red dots mark the moments when buckling occurred. (A2, B2, C2) Same parameters as in experiment, but now nondimensional and plotted against the scaled time following eq 4 . The data collapse clearly shows the universality of the drop evaporation process. (A2) The final volume is controlled by the occurrence of crystallization, rather than by the liquid-vapor equilibrium relation, which is shown by the black dashed line predicted by eq 6 .
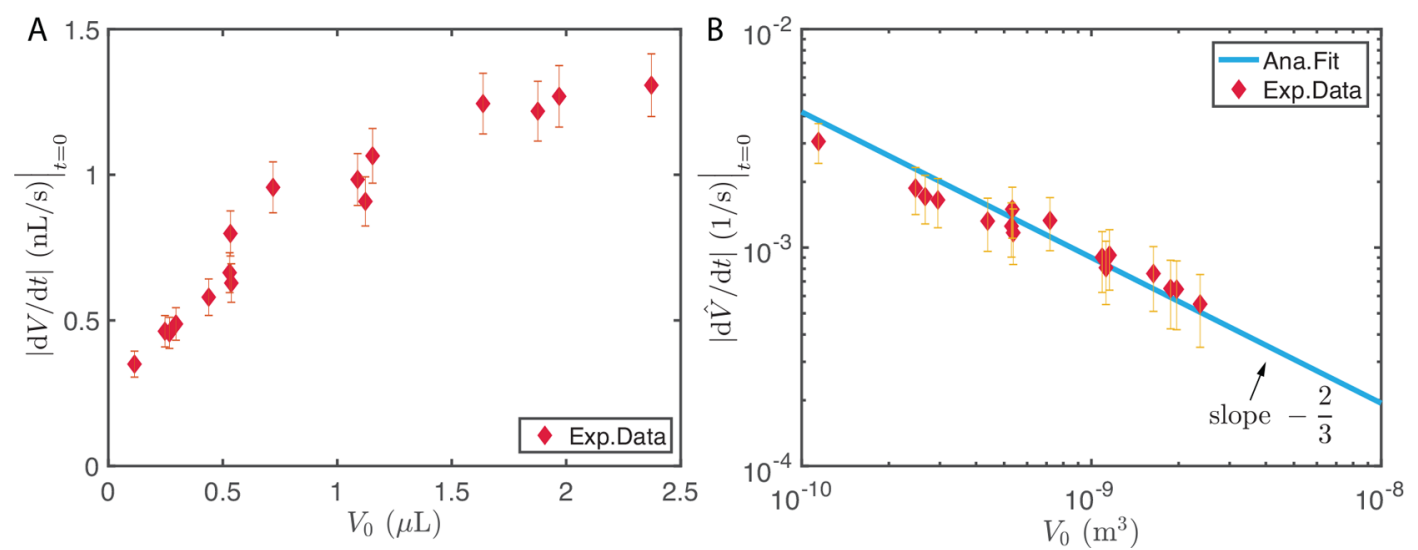

Figure 4. (A) Initial rate of volume loss of the drop varies for different initial volumes. (B) Same data normalized by the initial volume is plotted against the initial volume. The straight line shows the scaling relation with slope $-2 / 3$, which is predicted by eq 5 , demonstrating good agreement with the experimental data. 


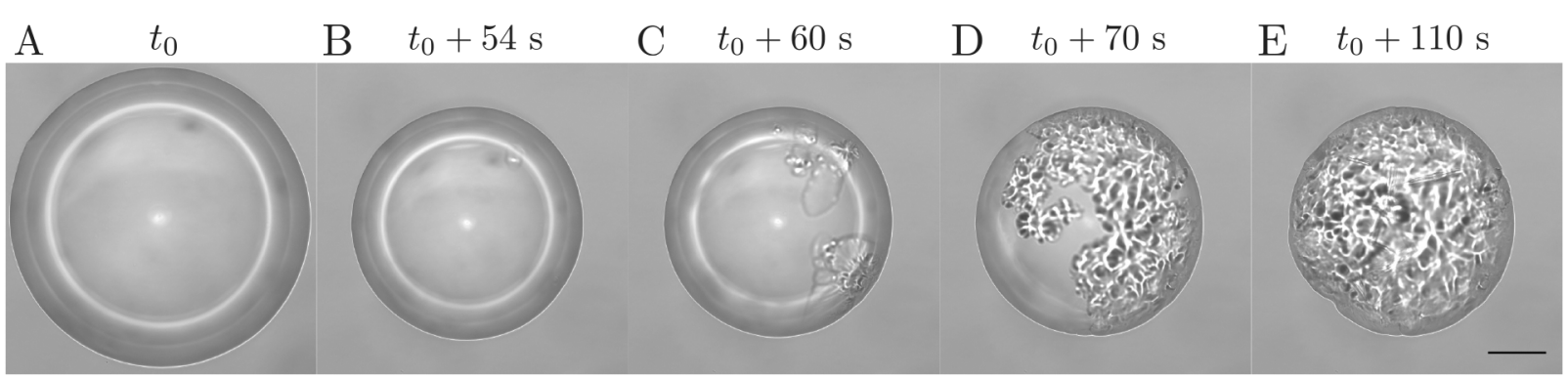

Figure 5. Bottom view of a complete drop lifetime (see Movie S1). (A) The drop evaporates on the substrate with receding contact line. (B) The first crystal appears near the contact line region. (C) Several crystals nucleate and grow independently. (D) Growing crystals coalesce with neighboring ones. (E) The crystals cover the whole drop and bring the evaporation to an end. (B-E) The contact line basically remains the same until the final state of the drop but slightly deforms due to the buckling of the drop surface. The scale bar represents $50 \mu \mathrm{m}$.
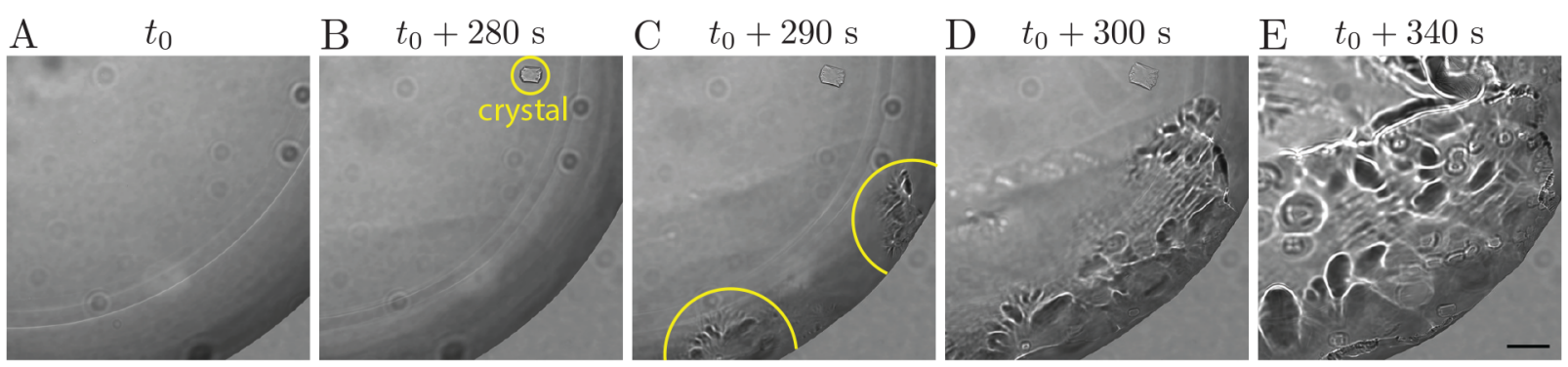

Figure 6. Bottom-view snapshots of the contact region of an evaporating surfactant-binary drop (see Movie S2). (A) The moment of deposition of the drop: the drop starts evaporating on the substrate. (B) A small crystal nucleates (yellow circle), floats, and grows near the contact line. (C) The crystals heterogeneously nucleate at the contact line. (D) The nucleated crystals grow and merge with neighboring crystals. (E) The crystallized SDS fully occupies the drop and eventually brings the evaporation to an end. The scale bar represents $20 \mu \mathrm{m}$.

monotonically increases with increasing droplet size, apart from fluctuations due to experimental uncertainties.

Crystallization Phase. Figure 5 shows the complete crystallization process of an evaporating surfactant-laden mixture droplet (see Movie S1). The droplet starts to evaporate at time $t_{0}$. At $\sim 50 \mathrm{~s}$, the first crystals appear near the contact line (CL) region. After a few more seconds, several crystals nucleate at the rim. Then they grow and coalesce to form a larger piece and finally occupy the whole bulk of the droplet. Figure 6 presents a zoomed-in bottom view of the contact region of another evaporating surfactant-laden mixture droplet. Initially, the droplet is transparent with a smooth CL. After evaporating for $280 \mathrm{~s}$, a crystal nucleates near the CL and floats to the position labeled by the yellow circle. A few seconds later, more crystals nucleate at the rim, slightly deforming the CL. The nucleated crystals grow and coalesce with neighboring crystals. Eventually, the whole droplet is occupied by the crystals, and the CL deforms and is no longer smooth. Figure 7A shows the temporal evolution of the transformed fraction measured in a $2 \mathrm{D}$ bottom view for three different droplets. $X$ is the area fraction occupied by crystals, and $t$ is the time which has elapsed after the first crystallization had been observed. The area fraction $X$ increases as the growth of crystals at a different rate for each droplet.

\section{THEORETICAL ANALYSIS}

Theory of Mixture Droplet Evaporation. We first study the evaporation characteristics of the surfactant-laden mixture droplet. In general, for a droplet evaporating on a flat surface under ambient conditions and in the absence of any correction, the evaporation is fully controlled by the diffusion of the vapor away from the droplet: ${ }^{26,27}$ the liquid molecules change their phase and diffuse as vapor molecules into the surrounding air.

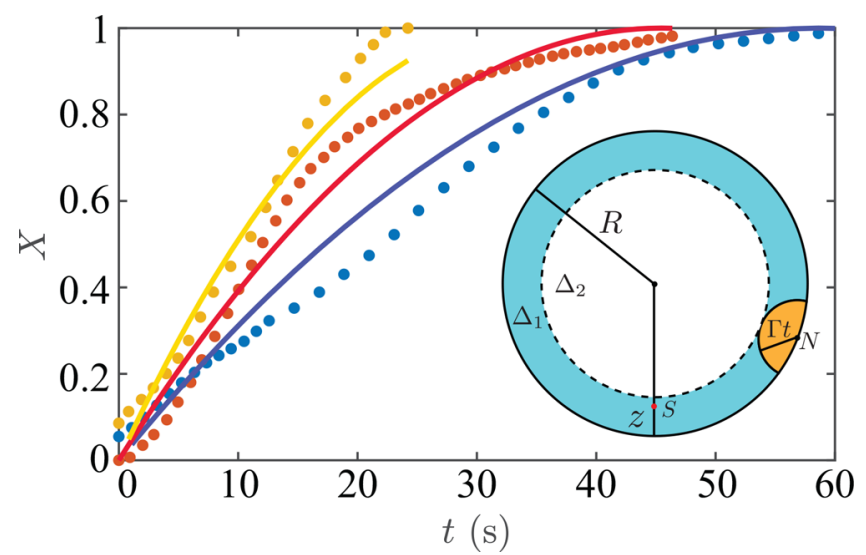

Figure 7. Measurement of the crystallized area fraction of three droplets against time. The analytical results (solid lines) according to eq 7 are also shown. (inset) The circular regions with areas $\Delta_{1}$ and $\Delta_{2}$ represent the areas where nucleation can or cannot occur in time $t$, respectively. The orange region indicates the area transformed at time $t$, due to a nucleation at N. $S$ is an arbitrary point in $\Delta_{1}$ which has distance $z$ from the boundary. Within time $t$, it must be transformed due to a nucleus on the boundary.

Popov $^{28}$ derived an analytical solution by using the solution of the equivalent problem of an electric potential around a charged lens-shaped conductor:

$$
\frac{\mathrm{d} m}{\mathrm{~d} t}=-\pi D R\left(c_{\mathrm{s}}-c_{\infty}\right) f(\theta)
$$

with 


$$
f(\theta)=\frac{\sin (\theta)}{1+\cos (\theta)}+4 \int_{0}^{\infty} \frac{1+\cosh (2 \theta \varepsilon)}{\sinh (2 \pi \varepsilon)} \tanh [(\pi-\theta) \varepsilon] \mathrm{d} \varepsilon
$$

with $m$ the droplet mass, $D$ the diffusion coefficient of the droplet liquid vapor in air, $c_{\mathrm{s}}$ the saturated concentration of liquid vapor molecules, and $c_{\infty}$ the ambient concentration of the liquid vapor far away from the drop.

For the evaporation of multicomponent droplets, we first employ the method suggested by Brenn, ${ }^{29}$ namely considering the total evaporation rate of the mixture droplet as the sum of the evaporation rate of each individual component. In our surfactant-laden glycerol-water droplet, glycerol and SDS are nonvolatile under ambient conditions. ${ }^{30}$ Therefore, only the diffusive flux of water contributes to the total evaporation rate. The essential difference between the evaporation of pure droplets and multicomponent droplets is the vapor-liquid equilibrium: the nonvolatile component in the system alters the saturated concentration of water vapor at the interface. ${ }^{18,31}$ Raoult's law $^{32}$ is used to calculate the saturated water vapor concentration of the binary system: $c_{\mathrm{w}, \mathrm{s}}=X_{\mathrm{w}} c_{\mathrm{w}, \mathrm{s}}^{0}$, where $X_{\mathrm{w}}$ is the mole fraction of water at the interface and $c_{\mathrm{w}, s}^{0}$ is the saturated vapor concentration of pure water. However, Raoult's law relies on an idealized solution and as such ignores any interaction between the components. To overcome this limitation, the so-called activity coefficient $\psi^{33}$ was introduced to describe this interaction. In our case, it specifically addresses the interaction between water and the other components: $c_{\mathrm{w}, s}=$ $\psi_{\mathrm{w}} X_{\mathrm{w}} c_{\mathrm{w}, s}^{0}$. By using the water activity coefficient $\psi_{\mathrm{w}}{ }^{34}$ in the modified Raoult's law, we obtain a theoretical model to express the evaporation rate for the binary droplet:

$$
\frac{\mathrm{d} m}{\mathrm{~d} t}=-\pi D R\left(\psi_{\mathrm{w}} X_{\mathrm{w}} c_{\mathrm{w}, \mathrm{s}}^{0}-c_{\mathrm{w}, \infty}\right) f(\theta)
$$

There is, however, one added complexity in our system: it is difficult to determine the exact $c_{\mathrm{w}, \mathrm{s}}$ without knowing the exact mole fraction of water, glycerol, and SDS molecules. Hence we cannot analytically predict the evaporation rate for each time instant. To compare different sets of experimental data, we rescale the measured droplet volume and time by introducing the nondimensional volume $\hat{V}=V / V_{0}$ and time $\hat{t}=t / \tau_{\mathcal{c}}$ with $V(t)$ the measured droplet volume and $V_{0}$ its initial volume. $\tau_{c}$ is the characteristic time scale of the diffusive evaporation, ${ }^{25,35}$ which can also be read from eq 1 :

$$
\tau_{c}=\frac{\rho R_{0}^{2}}{D \Delta c}
$$

Figures $3 \mathrm{~A} 2, \mathrm{~B} 2, \mathrm{C} 2$ show that the rescaled experimental data for volume $V / V_{0}$, contact angle $\theta$, and footprint radius $R / R_{0}$ versus the dimensionless time $t / \tau_{\mathrm{c}}$ follow a universal curve for all measured droplet sizes. The collapse of all the curves demonstrates that regardless of the initial size, the droplets with the same initial composition always follow the same evaporation behavior, with a universal evolution of all geometrical characteristics. On the basis of this, we can conclude that the variations of not only the geometry but also the internal composition concentration and distribution are universal, independent of the droplet size.

We also compare the initial evaporation rate of different initial volumes by introducing the dimensionless volume loss rate $\mathrm{d} \hat{V} / \mathrm{d} t=\mathrm{d}\left(V / V_{0}\right) / \mathrm{d} t$. According to eq 3 , the dimensionless initial evaporation rate is

$$
\left.\frac{\mathrm{d} \hat{V}}{\mathrm{~d} t}\right|_{t=0} \propto \frac{D \Delta c R_{0}}{\rho V_{0}} \propto \frac{D \Delta c}{\rho V_{0}^{2 / 3}}
$$

On the basis of the $V_{0}^{-2 / 3}$ proportionality of eq 5, we rescale the experimental data of Figure 4A (see Figure 4B) and plot them on a double-logarithmic scale. Indeed, the data follow the scaling law as suggested by eq 5 , confirming our model assumptions.

Besides controlling the evaporation rate, the model also yields the terminal state of the evaporation, which is when the saturated water vapor concentration equals the environmental concentration, $c_{\mathrm{w}, \mathrm{s}}=c_{\mathrm{w}, \infty}$. Essentially, the evaporation stops when the active mole fraction of water equals the relative humidity $H$ of the surrounding air, $\psi_{\mathrm{w}} X_{\mathrm{w}}=H$. For the same reason as mentioned above, we only compare the experimental data with the analytical prediction for glycerol-water binary system, ignoring the mole fraction of the surfactant. From the relative humidity $H$ measured in experiment, we can calculate analytically the "theoretical final volume" $V_{\mathrm{t}}$ (see Supporting Information) as

$$
V_{\mathrm{t}}=\left(\frac{M_{\mathrm{w}}}{M_{\mathrm{g}}} \frac{H}{\psi_{\mathrm{w}}-H}+\frac{\rho_{\mathrm{w}}}{\rho_{\mathrm{g}}}\right)\left(\frac{1-C_{\mathrm{g}}}{C_{\mathrm{g}}}+\frac{\rho_{\mathrm{w}}}{\rho_{\mathrm{g}}}\right)^{-1} V_{0}
$$

where $M_{\mathrm{g}}=9.21 \times 10^{-2} \mathrm{~kg} / \mathrm{mol}$ and $M_{\mathrm{w}}=1.8 \times 10^{-2} \mathrm{~kg} / \mathrm{mol}$ are the molecular mass of glycerol and water, respectively, $\rho_{\mathrm{g}}=$ $1.226 \times 10^{3} \mathrm{~kg} / \mathrm{m}^{3}$ and $\rho_{\mathrm{w}}=0.997 \times 10^{3} \mathrm{~kg} / \mathrm{m}^{3}$ are their liquid densities at room temperature, and $C_{\mathrm{g}}$ is the initial mass concentration of glycerol in each measurement. The final volume of the equilibrium state (dashed line in Figure 3A2) lies below the final volumes of all the droplets, which indicates that the shielding of water by the crystallized interface blocked any further evaporation before the system reached its equilibrium state.

Theory of 2-Dimensional Finite System Crystallization. As it is well-known, the evaporation rate has a singularity at the rim of the droplet, provided the contact angle is smaller than $90^{\circ}, 6,18$ which in our system, where the contact angle ranges from $65^{\circ}$ to $40^{\circ}$ during the evaporation process, indeed is the case. The singularity implies that the water depletes the fastest at the rim, which locally leads to a higher concentration of glycerol at that part. It is therefore also expected that crystal nucleation occurs first near the $C L$ region due to the highest degree of oversaturation of SDS.

To model the crystallization, we employ a 2-dimensional model which is derived by extending the JMAK formalism ${ }^{36-40}$ to a finite 2-dimensional system with nonuniform nucleation. Based on the spherical shape of the droplet, the footprint area is circular and the nucleation starts near the contact line. We assume that the crystallization process occurs within a circular region, and nucleation is permitted at $t=0$ at various points on the perimeter of the area. Figure $7 \mathrm{~B}$ shows the geometry of the two regions $\Delta_{1}$ and $\Delta_{2}$ within a circle with radius $R$ (drop radius): the $\Delta_{2}$ region is completely free of crystallization, while $\Delta_{1}$ is partially crystalline. The maximum growth radius of the crystals is given by $\Gamma t$, where $\Gamma$ is the constant growth rate. Weinberg ${ }^{41,42}$ proposed an analytical model to describe the fraction $X(t)$ transformed at time $t$, namely

$$
X(t)=\left[1-(1-\Gamma t / R)^{2}\right] X_{1}(t)
$$

where $X_{1}(t)$ represents the fraction which has crystallized in $\Delta_{1}$. It can be expressed as ${ }^{42}$ 


$$
\begin{aligned}
& X_{1}(t)=1-\int_{1-y}^{1} \exp \left[-2 P_{1} R \cos ^{-1}\left(\frac{1+\Phi^{2}-y^{2}}{2 \Phi}\right)\right] \Phi \mathrm{d} \Phi \\
& \quad \times \frac{1}{2}\left[1-(1-\Phi)^{2}\right]^{-1}
\end{aligned}
$$

with $\Phi=(R-z) / R$ and $y=\Gamma t / R . z$ denotes the distance between an arbitrary point $S$ in the $\Delta_{1}$ region and the boundary. $P_{1}$ is the nucleation probability per unit length in region $\Delta_{1}$.

We demonstrate that the transformation rate is more sensitive to the growth rate $\Gamma$ rather than to the seeding probability $P_{1}$, as shown in Supporting Information. Here we set $P_{1}=1000 \mu \mathrm{m}^{-1}$ by assuming a saturated nuclei density. We test this theory for the three cases in Figure 7A with droplet footprint radius $R_{1}=146 \mu \mathrm{m}, R_{2}=110 \mu \mathrm{m}$, and $R_{3}=86 \mu \mathrm{m}$. By fitting the theoretical curves to the experimental data, we obtain the growth rate as the fitting parameter: Quite consistently, the results are $\Gamma_{1}=2.48 \mu \mathrm{m} / \mathrm{s}, \Gamma_{2}=2.42 \mu \mathrm{m} / \mathrm{s}$, and $\Gamma_{3}=2.58 \mu \mathrm{m} / \mathrm{s}$ for the three analyzed cases. From Figure $6 \mathrm{C}$, we estimate the crystal growth rate in the early crystallization stage by measuring the increasing rate of crystal size near the contact line within the yellow circle. We obtain the estimate $\Gamma \approx 25 \pm 5 \mu \mathrm{m} / 10 \mathrm{~s}=2.5 \pm 0.5 \mu \mathrm{m} / \mathrm{s}$, which is comparable to the values $\Gamma_{1}, \Gamma_{2}$, and $\Gamma_{3}$ obtained from our model. Even though we applied a $2 \mathrm{D}$ model to a $3 \mathrm{D}$ problem, the theoretical predictions show good agreement with experimental data: the reason is that our droplet is relatively flat, with a contact angle of about $40^{\circ}$ when crystallization occurs.

\section{CONCLUSIONS}

In summary, crystallization of sodium dodecyl sulfate induced by selective evaporation in a surfactant-laden glycerol-water mixture droplet is observed during the evaporation process. We studied experimentally the dynamics of evaporation prior to the occurrence of crystal nucleation and the kinetics of crystallization, thereafter. We applied a diffusion model extended by Raoult's law to describe the evaporation characteristics and could reveal a universal evaporation behavior, independent of the size of the droplets. Finally, we applied a 2-dimensional model building on the JMAK nucleation model to describe the kinetics of the crystallization. Thanks to the low contact angle, this model can successfully describe our experimental data on nucleation.

Surfactants attract significant attention as their ubiquitous role in fluid dynamics of either nature or technology. ${ }^{43}$ Our findings clearly show an unexpected consequence of using surfactants in such evaporating systems. This particularly holds for inkjet printing where surfactants are extensively used. As nearly all inks contain various components with different volatilities, the variations of the composition ratio caused by the selective evaporation of more volatile components may lead to the segregation of surfactants in the form of liquid phase separation $^{14}$ or crystallization. Our study may rise the awareness of using surfactants with cautions in such multicomponent systems, which normally involves rich physicochemical processes. ${ }^{44}$

Some issues remain open and unexplored. As the temperature can change the CMC of SDS in glycerol-water mixture, ${ }^{45}$ does the crystallization behavior also depend on the temperature? How to describe the buckling behavior after the occurrence of crystallization? Another question is on the morphology of the SDS crystals; e.g., is the crystal structure different from the one induced upon cooling? Such questions are of great interest in view of crystal chemistry, and it is worthwhile to further investigate such crystallization behavior from a microscopic perspective in the future.

\section{ASSOCIATED CONTENT}

\section{Supporting Information}

The Supporting Information is available free of charge at https://pubs.acs.org/doi/10.1021/acs.langmuir.0c01169.

Derivation of the theoretical final volume, the image analysis for crystallization process, and the discussion of JMAK model (PDF)

Movie S1: the crystallization process from the bottom of the droplet (MOV)

Movie S2: the crystallization process in the contact line region of the droplet (MP4)

\section{AUTHOR INFORMATION}

\section{Corresponding Author}

Detlef Lohse - Physics of Fluids Group, Department of Science and Technology, Mesa+ Institute for Nanotechnology, Max Planck Center for Complex Fluid Dynamics and J. M. Burgers Centre for Fluid Dynamics, University of Twente, 7500 AE Enschede, The Netherlands; Max Planck Institute for Dynamics and Self-Organization, 37077 Göttingen, Germany;

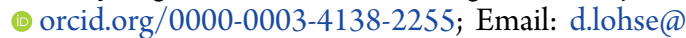
utwente.nl

\section{Authors}

Yaxing Li - Physics of Fluids Group, Department of Science and Technology, Mesa+ Institute for Nanotechnology, Max Planck Center for Complex Fluid Dynamics and J. M. Burgers Centre for Fluid Dynamics, University of Twente, 7500 AE Enschede, The Netherlands; 10 orcid.org/0000-0003-1318-7073

Valentin Salvator - Physics of Fluids Group, Department of Science and Technology, Mesa+ Institute for Nanotechnology, Max Planck Center for Complex Fluid Dynamics and J. M. Burgers Centre for Fluid Dynamics, University of Twente, 7500 AE Enschede, The Netherlands

Herman Wijshoff - Department of Mechanical Engineering, Eindhoven University of Technology, 5600 MB Eindhoven, The Netherlands; Oće Technologies B.V., 5900 MA Venlo, The Netherlands

Michel Versluis - Physics of Fluids Group, Department of Science and Technology, Mesa+ Institute for Nanotechnology, Max Planck Center for Complex Fluid Dynamics and J. M. Burgers Centre for Fluid Dynamics, University of Twente, 7500 AE Enschede, The Netherlands; 10 orcid.org/0000-00022296-1860

Complete contact information is available at: https://pubs.acs.org/10.1021/acs.langmuir.0c01169

\section{Notes}

The authors declare no competing financial interest.

\section{ACKNOWLEDGMENTS}

We thank Shuai Li for valuable suggestions on the manuscript. This work is part of an Industrial Partnership Programme (IPP) of The Netherlands Organization for Scientific Research (NWO). This research programme is cofinanced by Canon Production Printing Netherlands B.V., University of Twente, 
and Eindhoven University of Technology. D.L. gratefully acknowledges support by his ERC-Advanced Grant DDD (project number 740479).

\section{REFERENCES}

(1) Sempels, W.; De Dier, R.; Mizuno, H.; Hofkens, J.; Vermant, J. Auto-production of biosurfactants reverses the coffee ring effect in a bacterial system. Nat. Commun. 2013, 4, 1757.

(2) Marin, A.; Liepelt, R.; Rossi, M.; Kähler, C. J. Surfactant-driven flow transitions in evaporating droplets. Soft Matter 2016, 12, 15931600.

(3) Kwieciński, W.; Segers, T.; van der Werf, S.; van Houselt, A.; Lohse, D.; Zandvliet, H. J. W.; Kooij, S. Evaporation of Dilute Sodium Dodecyl Sulfate Droplets on a Hydrophobic Substrate. Langmuir 2019, 35, 10453-10460.

(4) Park, J.; Moon, J. Control of colloidal particle deposit patterns within picoliter droplets ejected by ink-jet printing. Langmuir 2006, 22, 3506-3513.

(5) Kong, Y. L.; Tamargo, I. A.; Kim, H.; Johnson, B. N.; Gupta, M. K.; Koh, T.; Chin, H.; Steingart, D. A.; Rand, B. P.; McAlpine, M. C. 3D Printed Quantum Dot Light-Emitting Diodes. Nano Lett. 2014, 14, 7017-7023.

(6) Deegan, R. D.; Bakajin, O.; Dupont, T. F.; Huber, G.; Nagel, S. R.; Witten, T. A. Capillary flow as the cause of ring stains from dried liquid drops. Nature 1997, 389, 827-829.

(7) Still, T.; Yunker, P. J.; Yodh, A. G. Surfactant-Induced Marangoni Eddies Alter the Coffee-Rings of Evaporating Colloidal Drops. Langmuir 2012, 28, 4984-4988.

(8) Kim, H.; Boulogne, F.; Um, E.; Jacobi, I.; Button, E.; Stone, H. A. Controlled uniform coating from the interplay of Marangoni flows and surface-adsorbed macromolecules. Phys. Rev. Lett. 2016, 116, 124501.

(9) Piret, J.; Desormeaux, A.; Bergeron, M. Sodium Lauryl Sulfate, a Microbicide Effective Against Enveloped and Nonenveloped Viruses. Curr. Drug Targets 2002, 3, 17-30.

(10) Kumar, A.; Bhattacharjee, G.; Kulkarni, B. D.; Kumar, R. Role of Surfactants in Promoting Gas Hydrate Formation. Ind. Eng. Chem. Res. 2015, 54, 12217-12232.

(11) Choudhary, N.; Hande, V. R.; Roy, S.; Chakrabarty, S.; Kumar, R. Effect of Sodium Dodecyl Sulfate Surfactant on Methane Hydrate Formation: A Molecular Dynamics Study. J. Phys. Chem. B 2018, 122, 6536-6542.

(12) Smith, L.; Duncan, A.; Thomson, G.; Roberts, K.; Machin, D.; McLeod, G. Crystallisation of sodium dodecyl sulphate from aqueous solution: phase identification, crystal morphology, surface chemistry and kinetic interface roughening. J. Cryst. Growth 2004, 263, 480490.

(13) Summerton, E.; Zimbitas, G.; Britton, M.; Bakalis, S. Crystallisation of sodium dodecyl sulfate and the corresponding effect of 1-dodecanol addition. J. Cryst. Growth 2016, 455, 111-116.

(14) Li, Y.; Lv, P.; Diddens, C.; Tan, H.; Wijshoff, H.; Versluis, M.; Lohse, D. Evaporation-Triggered Segregation of Sessile Binary Droplet. Phys. Rev. Lett. 2018, 120, 224501.

(15) Kim, H.; Stone, H. Direct measurement of selective evaporation of binary mixtures by dissolving materials. J. Fluid Mech. 2018, 850, 769.

(16) Li, Y.; Diddens, C.; Lv, P.; Wijshoff, H.; Versluis, M.; Lohse, D. gravitational effect in evaporating binary microdroplets. Phys. Rev. Lett. 2019, 122, 114501.

(17) Vitale, S. A.; Katz, J. L. Liquid droplet dispersions formed by homogeneous liquid-liquid nucleation: "The ouzo effect. Langmuir 2003, 19, 4105-4110.

(18) Tan, H.; Diddens, C.; Lv, P.; Kuerten, J. G. M.; Zhang, X.; Lohse, D. Evaporation-triggered microdroplet nucleation and the four life phases of an evaporating Ouzo drop. Proc. Natl. Acad. Sci. U. S. A. 2016, 113, 8642-8647.
(19) Tan, H.; Diddens, C.; Versluis, M.; Butt, H.-J.; Lohse, D.; Zhang, X. Self-wrapping of an Ouzo Drop Induced by Evaporation on a Superamphiphobic Surface. Soft Matter 2017, 13, 2749-2759.

(20) Mailleur, A.; Pirat, C.; Pierre-Louis, O.; Colombani, J. Hollow Rims from Water Drop Evaporation on Salt Substrates. Phys. Rev. Lett. 2018, 121, 214501.

(21) Peng, S.; Xu, C.; Hughes, T. C.; Zhang, X. From Nanodroplets by the Ouzo Effect to Interfacial Nanolenses. Langmuir 2014, 30, 12270-12277.

(22) van der Bos, A.; van der Meulen, M.-J.; Driessen, T.; van den Berg, M.; Reinten, H.; Wijshoff, H.; Versluis, M.; Lohse, D. Velocity Profile inside Piezoacoustic Inkjet Droplets in Flight: Comparison between Experiment and Numerical Simulation. Phys. Rev. Appl. 2014, 1, No. 014004.

(23) Cazabat, A. M.; Guéna, G. Evaporation of macroscopic sessile droplets. Soft Matter 2010, 6, 2591-2612.

(24) Stauber, J.; Wilson, S.; Duffy, B.; Sefiane, K. On the lifetimes of evaporating droplets. J. Fluid Mech. 2014, 744, R2.

(25) Lohse, D.; Zhang, X. Surface nanobubble and surface nanodroplets. Rev. Mod. Phys. 2015, 87, 981-1035.

(26) Picknett, R. G.; Bexon, R. The evaporation of sessile or pendant drops in still air. J. Colloid Interface Sci. 1977, 61, 336-350.

(27) Hu, H.; Larson, R. G. Marangoni Effect Reverses Coffee-Ring Depositions. J. Phys. Chem. B 2006, 110, 7090-7094.

(28) Popov, Y. O. Evaporative deposition patterns: Spatial dimensions of the deposit. Phys. Rev. E 2005, 71, No. 036313.

(29) Brenn, G.; Deviprasath, L.; Durst, F.; Fink, C. Evaporation of acoustically levitated multi-component liquid droplets. Int. J. Heat Mass Transfer 2007, 50, 5073-5086.

(30) Geballe, M. T.; Skillman, A. G.; Nicholls, A.; Guthrie, J. P.; Taylor, P. J. The SAMPL2 blind prediction challenge: introduction and overview. J. Comput.-Aided Mol. Des. 2010, 24, 259-279.

(31) Diddens, C.; Kuerten, J.; van der Geld, C.; Wijshoff, H. Modeling the evaporation of sessile multi-component droplets. $J$. Colloid Interface Sci. 2017, 487, 426-436.

(32) Raoult, F.-M. Loi générale des tensions de vapeur des dissolvants. Comptes Rendus 1887, 104, 1430-1433.

(33) Chu, S.; Prosperetti, A. Dissolution and growth of a multicomponent drop in an immiscible liquid. J. Fluid Mech. 2016, 798, 787-811.

(34) Marcolli, C.; Peter, T. Water activity in polyol/water systems: new UNIFAC parameterization. Atmos. Chem. Phys. 2005, 5, 15451555.

(35) Gelderblom, H.; Marin, A. G.; Nair, H.; van Houselt, A.; Lefferts, L.; Snoeijer, J. H.; Lohse, D. How water droplets evaporate on a superhydrophobic substrate. Phys. Rev. E 2011, 83, No. 026306.

(36) Avrami, M. Kinetics of Phase Change. I General Theory. J. Chem. Phys. 1939, 7, 1103.

(37) Avrami, M. Kinetics of Phase Change. II Transformation-Time Relations for Random Distribution of Nuclei. J. Chem. Phys. 1940, 8, 212

(38) Avrami, M. Granulation, Phase Change, and Microstructure Kinetics of Phase Change III. J. Chem. Phys. 1941, 9, 177.

(39) Johnson, W.; Mehl, R. Reaction kinetics in processes of nucleation and growth. Trans. AIME 1939, 135, 416.

(40) Kolmogorov, A. A statistical theory for the recrystallization of metals. Isz. Akad. Nauk SSR. Ser. Mater. 1937, 3, 355.

(41) Weinberg, M. C. Surface nucleated transformation kinetics in 2and 3-dimensional finite systems. J. Non-Cryst. Solids 1991, 134, 116122

(42) Weinberg, M. C.; Birnie, D. P.; Shneidman, V. A. Crystallization kinetics and the JMAK equation. J. Non-Cryst. Solids 1997, 219, 89-99.

(43) Manikantan, H.; Squires, T. M. Surfactant dynamics: hidden variables controlling fluid flows. J. Fluid Mech. 2020, 892, P1.

(44) Lohse, D.; Zhang, X. Physicochemical Hydrodynamics of Droplets out of Equilibrium. Nat. Rev. Phys. 2020, in press. 
(45) Ruiz, C. C.; Díaz-López, L.; Aguiar, J. Micellization of Sodium

Dodecyl Sulfate in Glycerol Aqueous Mixtures. J. Dispersion Sci.

Technol. 2008, 29, 266-273. 